\title{
Exoskeleton - wearable devices. Literature review
}

\author{
Marius-Leonard Olar $^{1}$, Monica Leba ${ }^{1,{ }^{*}}$, Marius Risteiu ${ }^{1}$ \\ ${ }^{1}$ University of Petroșani, România
}

\begin{abstract}
Exoskeletons are companion devices that help a person to perform various daily activities. These can range from work to medical rehabilitation. The type of activity performed depend on the construction and control of the exoskeleton, so that some devices are for only one arm, others for both, can be driven by motors directly or through cables. Exoskeletons can be driven based on information received from position, force, speed sensors or by using EMG, EEG signals. Exoskeleton wearable devices began to appear around 1980, as an aid in physical work, in the handling of various heavy objects. Over time, they also covered the preventive-rehabilitation medical side, in order to reduce muscle pain or to restore specific movements, attenuated or even missing following accidents or diseases of the muscles. The paper presents an overview of the exoskeletons developed for the human arm.
\end{abstract}

Keywords: bio-inspired, assistive devices, wearable robots, workload

\section{Introduction}

This article is a study of wearable exoskeleton devices for the upper limb. Since it interacts mechanically with the musculoskeletal structure of the arm, it must take over, as much as possible, its shape and functionality, depending on the needs for which it was designed [1]. These devices are associated with several purposes, including medical rehabilitation, in the case of neuromuscular injuries, assistance in an industrial environment, amplification of power, or support for persons with difficulties in moving or handling objects, in daily activities.

Even though man's first companion mechanism appeared in the 1960s [2], as a special equipment of the American armed forces, the real exoskeletons appeared on the researchers' agenda only in the 1980s, when the period of physical interaction between man and robot began. The initial purpose of the exoskeletons was to assist in physical work, in industry, in the handling of heavy objects [3], but in the passage of years this goal was also directed towards medical applications, potentiation of physical force, correction and support of movements of the lower and upper limbs.

Exoskeletons for the upper limb are divided into several categories, for the shoulder joint, for the elbow or for the wrist, taken independently or combined with each other. Those in

\footnotetext{
*Corresponding author: monicaleba@upet.ro
} 
charge of the design and development of these devices must combine knowledge of human anatomy, biomechanics, sensors and motor control with a design appropriate to the intended purpose, with kinematic chain control algorithms and others [4].

During this period, the design and functionality of the upper limb exoskeletons goes to the biomedical side, helping the elderly or disabled, who, with the help of sensors, artificial intelligence and virtual or augmented projection, can become true rehabilitation nurses [5].

\section{Hardware design of exoskeletons}

An exoskeleton is an inferior system attached to a superior system, in this case, the human arm. In this complex system the inferior system must copy, as much as possible, all the capabilities of the superior system, or as many as possible, and then be able to reproduce them. By the possibility of reproducing the capabilities of the superior system, the inferior one can control and rehabilitate, in the event of malfunctions, the superior system. This control can "see" which of the elements of the second system needs to be assisted or improved. The human upper limb has 7 degrees of freedom (DoF), and a mechanized arm (an exoskeleton) can have only 6 degrees of freedom.

Table 1. Range of motion of each type of joint [6].

(A-Anatomical, E-Exoskeleton)

\begin{tabular}{|l|c|l|}
\hline \multicolumn{2}{|c|}{ Degrees of freedom } & \multicolumn{1}{|c|}{ Motion interval } \\
\hline Shoulder - Flexion/Extension & A-E & $150^{\circ}-180^{\circ} / 40^{\circ}-50^{\circ}$ \\
\hline Shoulder - Abduction/Adduction & A-E & $180^{\circ} / 30^{\circ}-40^{\circ}$ \\
\hline Shoulder - Internal/External Rotation & A-E & $70^{\circ}-95^{\circ} / 40^{\circ}-70^{\circ}$ \\
\hline Elbow - Flexion/Extension & A-E & $135^{\circ}-1400 / 0^{\circ}$ \\
\hline Forearm - Supination/Pronation & A-E & $85^{\circ}-90^{\circ} / 70^{\circ}-90^{\circ}$ \\
\hline Wrist - Flexion/Extension & A-E & $73^{\circ} / 70^{\circ}$ \\
\hline Wrist - Radial/Ulnar Deviation & A & $27^{\circ} / 27^{\circ}$ \\
\hline
\end{tabular}

These degrees of freedom help us to classify systems in conjunction with the number of elements of the system, which leads to a certain control protocol. This classification also takes into account the number of points at which the human arm comes into contact with the elements of an exoskeleton (a larger number may mean better control over the human arm, but also a certain resistance of the arm over the exoskeleton).

There is no particular standard in the design or functionality of an exoskeleton, in this case for the upper limb, but if we consider certain aspects of construction, actuation, transmission, sensors or control of the components, we can achieve an empirical classification of them [7]:

- Mechanism: can be for the entire arm, or for each independent element or taken two by two, with active or passive elements, for one arm or for both;

- Actuation: can be with electric, pneumatic, hydraulic motors;

- Transmission: with elements directly connected to motors, gears, artificial muscles or cables [8];

- Sensors: EMG, GEEG, or force/torque;

- Control: IMU, force/torque or impact. 
The design can be bio-inspirational (trying to reproduce human anatomical components, optimize their shapes and functionalities) [9], mechanically optimized, the exoskeleton can be fixed or wearable, soft or hard and can have applications in the fields: medical, for rehabilitation, industry or improvement of power/resistance performance, or assistant in daily work.

\subsection{Exoskeleton Operation}

The operation of exoskeleton elements is carried out by means of several types of actuators: electrical [10], pneumatic [11], hydraulic [12] or elastic [13, 14], each having advantages and disadvantages in terms of power, control, mobility or system maintenance. So, electric actuators give high control, less weight and the possibility of making a wearable exoskeleton, while pneumatic or hydraulic actuators give a high power-to-mass torque, to the detriment of portability and system maintenance (see Table 2).

Table 2. Types of exoskeleton actuators

\begin{tabular}{|c|c|c|c|c|c|}
\hline Motor type & Fixed/Wearable & Power & Control & Maintenance & Bio-inspirational \\
\hline Electric & $+/-$ & - & + & + & $+/-$ \\
\hline Pneumatic & $+/-$ & + & - & - & - \\
\hline Hydraulic & $+/-$ & + & - & - & - \\
\hline Elastic & $-/+$ & + & $+/-$ & - & + \\
\hline
\end{tabular}

\subsection{Transmission}

When designing an exoskeleton, there must be taken into account its ergonomics, in the sense that the resulting device does not have too much volume, is not too heavy or does not disturb the natural movement of the arm. Thus, the developers of these devices try different constructive variants to link the components together, using toothed wheels, direct links to the actuators, cables [15], Bowden cables [16], chains or elastic tendon slates [17, 18]. The disadvantage of the equipment with many elements is the oversizing of the gravitational compensators, which could reduce the level of effectiveness of human-robot interaction.

Cable transmission can lead to an ergonomic model of an exoskeleton. All heavy or bulky elements, such as actuators and controls, can be found on the back of the device, and light ones will compose the part that will come into contact with the user's arm. As disadvantages, can be mentioned: the need to use strong cables, to support the weight of the arm, to create optimal routes, to fluidly pass the cable, to avoid friction, to maintain cables to a certain degree of tension and the need to use several actuators [19].

\subsection{Detection Method}

The important characteristics of a system, such as an exoskeleton, are those related to detection and estimation. There is no need to deploy stand-alone sensors for speed, acceleration, torque or the positioning of component elements, because all of these are now integrated into electrical actuators.

By exploiting the data from various external sensors, the exoskeletons can have the following destinations: correcting a user's movements; assistance to become the support of a worker's arm while performing a hard work; performing a movement [20], from a 
foreshadowed scenario for a patient who does not have the ability to perform that movement [5]; to help a person lift and carry certain heavy objects; to make a movement generated by a patient's intention to move own arm [21]; or to be transformed into sports equipment, exerting certain physical resistances [22], leading to increased muscle mass.

\subsection{Control}

In order to perform the tasks for which it was designed, an exoskeleton must perform the required movements following sensor data processing. This data is processed by system controllers [23], which send the appropriate control signals to the actuators [24].

Control of the components refers to the change of position, speed, tightening force, dynamic change of behavior [25], by controlling impedance, adjusting the haptic feedback by which the fluidity of the movements of the components is regulated. A parental tree can also be structured, in which the human arm is master, and the exoskeleton arm is slave, to teleoperate the arm bound to the exoskeletal arm, of the mirror therapy type or to manipulate remotely in a rehabilitation treatment [24], via the internet, the exoskeleton of a patient, at home, is teleoperated by an assistant from the hospital [5]. By implementing Artificial intelligence, mechanical exoskeleton devices could take over and learn the behaviour of the human user in order to predict the movements the user would make [26].

\subsection{Biomechanics}

Studies on models of wearable exoskeleton devices for the human arm show the need for a biomechanical model [27], close to the natural, human model [28]. The human shoulder joint as a whole is a complex system, consisting of bones with a multitude of tendons connecting points, ligaments that passively bind the bones, four (or even five) types of joints and several important groups of muscles. In order to create and develop a wearable exoskeletal system that does not disturb or obstruct the movements of the wearer, researchers should consider the human musculoskeletal model, with the reproduction of essential elements and functionalities [28]. The product, in its bio-inspirational format, must have an optimized, compact mechanical design that is lightweight, durable, to become an "invisible" companion in the life of the wearer [30].

\section{Types of exoskeletons for the upper limb}

\subsection{Rehabilitation exoskeletons}

There are around 60,000 new cases of strokes in Romania each year. These strokes are one of the main causes of disability, and fall within the $60 \%$ of the causes of death, attributed to cardio-vascular diseases, with around 55,000 death cases yearly. Those who survive, in Romania there are around 250,000 people who survived a stroke, remain with a slight to severe weakness of the upper limbs. Affected people often lose their motor skills [31], including the ability to catch and lift, as well as shaking and abnormal coordination [32]. Medically, these conditions are given various physical therapies, with the help of which an attempt is made to improve the movements of the upper limbs in terms of strength, speed and coordination [33].

In the early 1980s, engineers and physical therapists attempted to automate these series of physical exercises by creating robotic systems, especially exoskeletons, to track the evolution of treatment and increase the number of types and repetitions. In the early years of these automated therapies, large exoskeletons were created and used, fixed in treatment 
rooms, which, over time, evolved towards reducing dimensions, but also towards the possibility that these exoskeletons would become wearable and as close to the human body as possible (see Table 3). These exoskeletons can execute multiple movements in degrees of freedom, somewhat close to those of the human arm, as well as the ability to control the power of the counterforce or compensate for the gravitational force [34].

Table 3. Types of exoskeletons used in the rehabilitation of upper limb functions

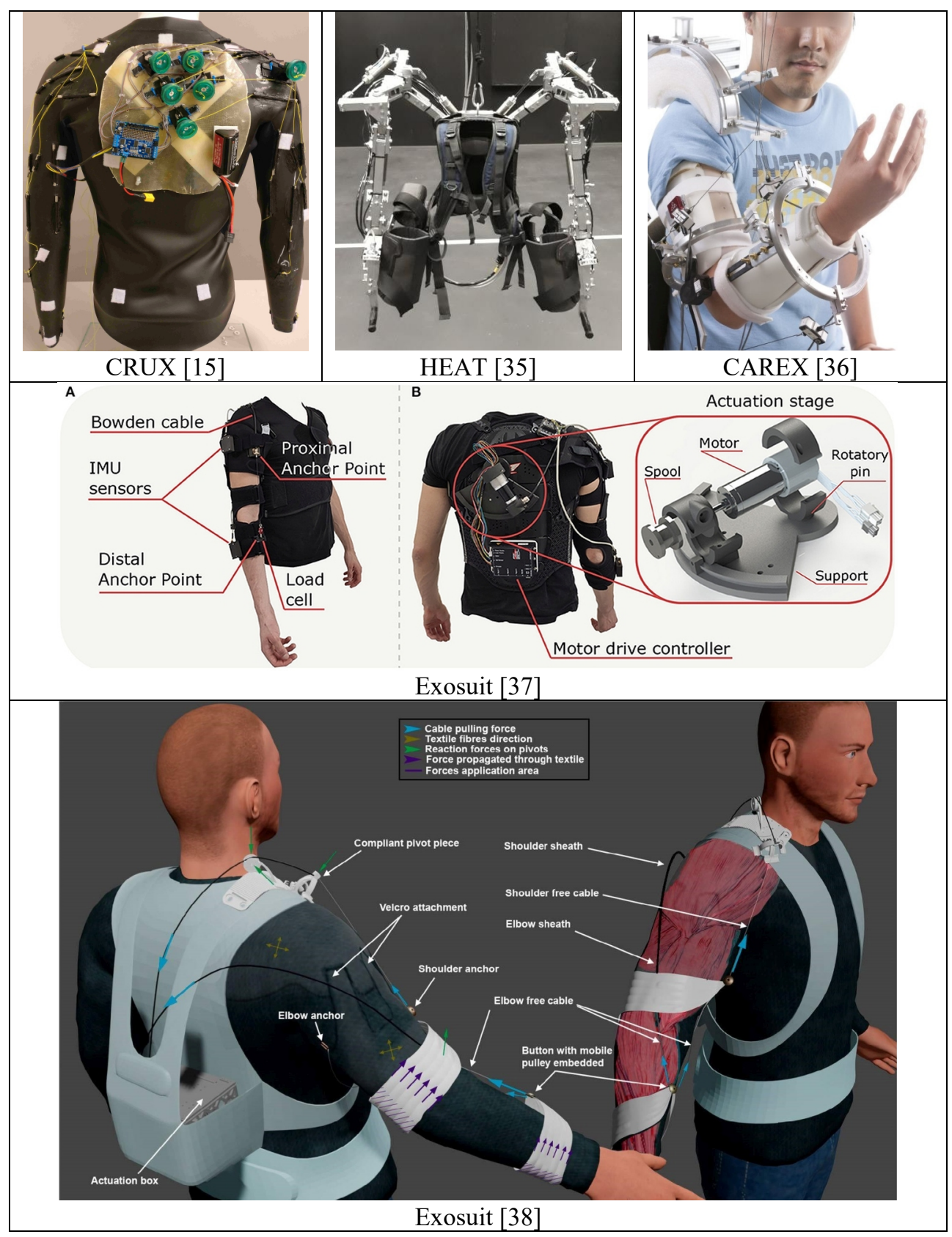




\subsection{Assistive exoskeletons}

Exoskeleton assist devices (see Table 4) are found between rehabilitation and augmentation devices. So, they can provide assistance in the rehabilitation treatments of patients who have suffered various injuries, affecting their muscles and implicitly the biomechanics of the upper limb, but also of healthy people who need, in the various daily work, an arm support (e.g. in an assembly work, in industry), or in a workout in the gym, and the exoskeleton device can replace the various sports equipment for increasing strength and resistance. They are also intended for people with muscle injuries, who also need devices in their daily activity.

This type of exoskeleton can provide passive support, limiting itself only to supporting the arm in a certain position for a certain period of time; active, triggered by the user's intention to execute a movement in a particular direction, or to complete the trajectory of a movement, to perform a task, or to force/guide the positioning of the arm on a particular trajectory (e.g. in performing the movements of some exercise correctly).

Table 4. Types of assistive exoskeletons in the activity of the upper limbs

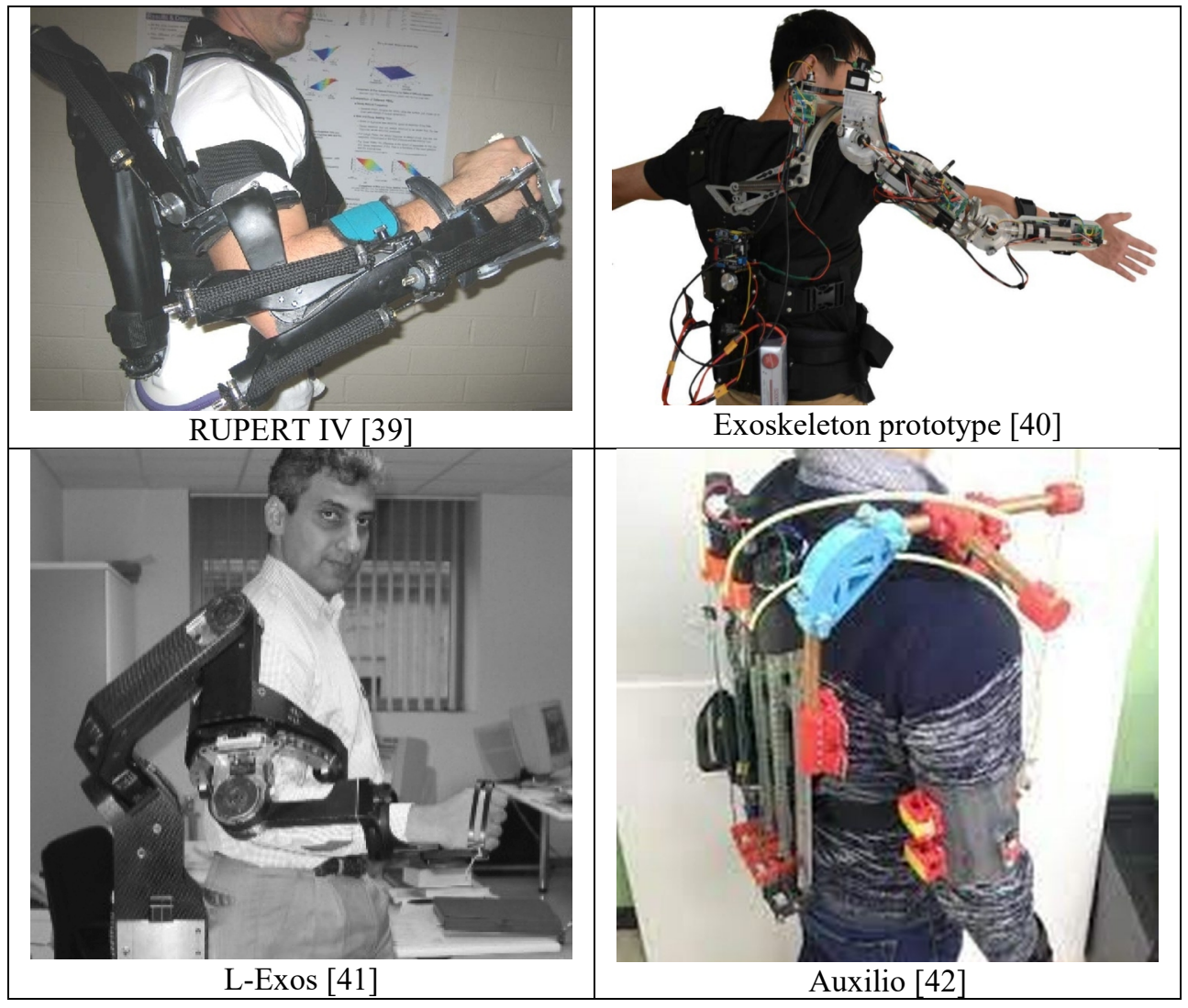

\subsection{Augmentation exoskeletons}

Augmentation exoskeletons are created and developed to improve human activity in heavy working conditions in industry, thus becoming a support in the work of users. Most are complete equipments, for the whole body, not just for certain parts, such as the upper or lower limbs. The subject, in this case, just drives that device, and all the weight of the manipulated 
objects is supported only by the exoskeleton. Other devices are just a tool for teleoperating robots in dangerous conditions for humans.

Table 5. Types of exoskeletons to increase human labor

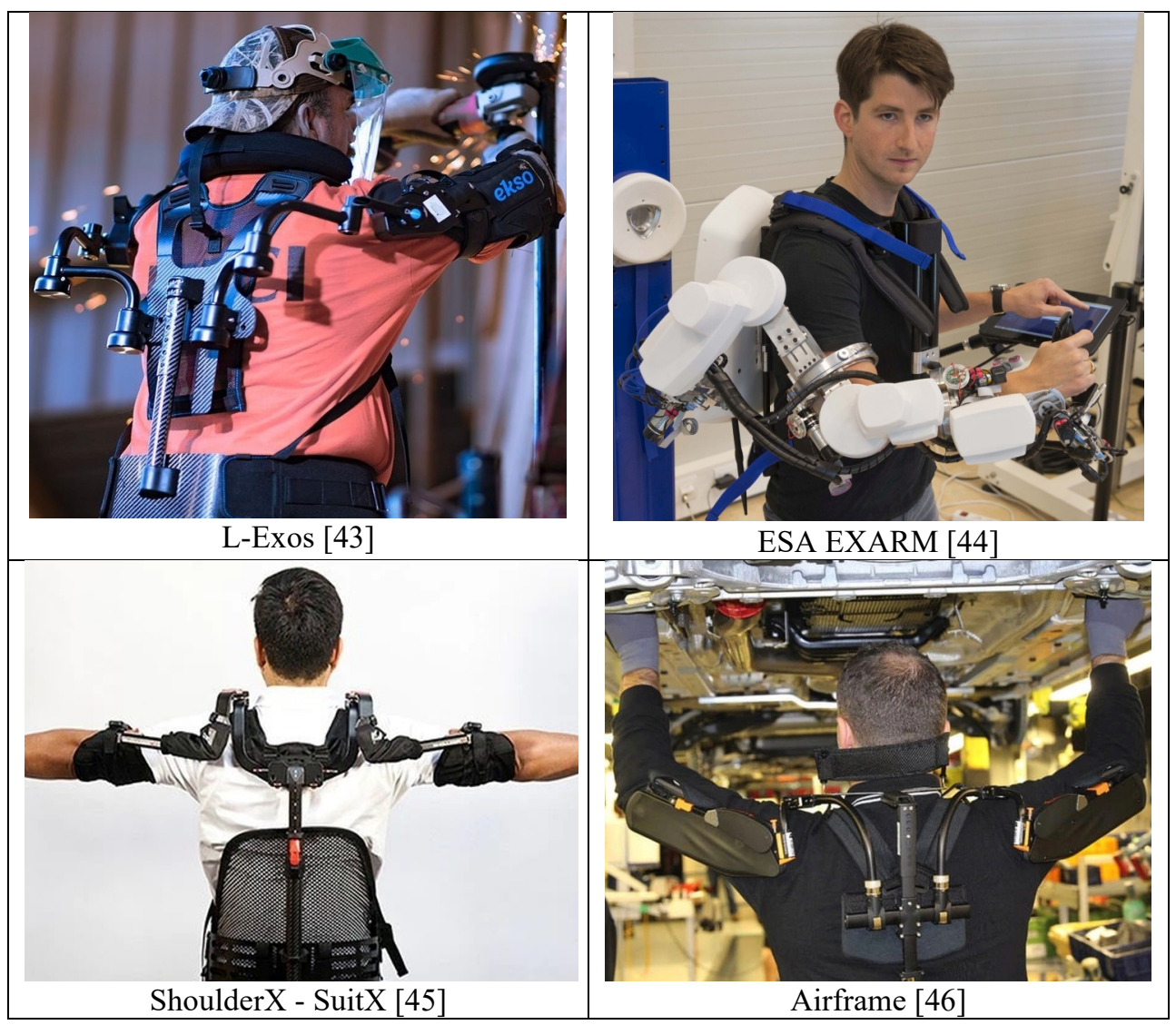

\section{Conclusions}

Exoskeleton systems for the upper limbs have a special value, given their applicability, from the medical rehabilitation of patients with various neuromuscular conditions, to companion in hard work, industry or physical training. This article provides an overview of the exoskeletons intended for the shoulder joint, in particular, of devices whose components are powered by electric motors, and the transmission is made by means of cables and those with a tendency towards biomimicry.

New challenges in the research and development of upper limb exoskeletons will lead to a design as close as possible to the human anatomical one, will be constructed from lightweight, powerful materials, and the tendency of researchers is to create wearable, autonomous devices that will become common in people's lives, such as clothing today.

\section{References}

1. KIM, Sunwook, et al. Applied ergonomics, (2018).

2. R.S. Mosher, Soc. Automot. Eng. Trans. 76 (1967).

3. M.N. Risteiu, M. Leba.W Conf. on Info Sys and Tech. (2020). 
4. M. Gunasekara, R. Gopura, S. Jayawardena, Int. J. Adv. Rob. Syst. 12 (2015).

5. M.L. Olar et al., MATEC Web of Conf. EDP Sciences, (2020).

6. Y. CHEN et al. Bio-med. mat. and eng., (2014).

7. Y. SHEN, P.W. FERGUSON, J. ROSEN, Wearable Robotics. Academic Press, (2020).

8. T.G. SUGAR et al. IEEE trans. on neural sys. and rehab. Eng., (2007).

9. N. JARRASSÉ et al. Frontiers in human neuroscience, (2014).

10. Ph. GARREC et al. In: 2008 IEEE/RSJ Inter. Conf. on Intell. Rob. and Sys. IEEE, (2008).

11. R.A.R.C.GOPURA, K. KIGUCHI, D.S.V. BANDARA, In: 2011 6th Inter. Conf. on Ind. and Inf. Sys. IEEE, (2011).

12. M. MISTRY, P. MOHAJERIAN, S. SCHAAL. In: 2005 IEEE/RSJ Inter. Conf. on Intel. Rob. and Sys. IEEE, (2005).

13. J. HURST, A. RIZZI, D. HOBBELEN. In: Inter. Conf. on climb. and walk. robots. (2004).

14. Z. LI, S. BAI. Mechan. and Mach. Th., (2019).

15. S. LESSARD et al. IEEE Trans. on Neural Sys. and Rehab. Eng., (2018).

16. P.W. FERGUSON et al. In: Inter. Symp. on Wearable Robotics. Springer, Cham, (2018).

17. C. JARRETT, A.J. MCDAID. IEEE Trans. on Neural Sys. and Rehab. Eng., (2017).

18. S. CREA et al. In: 2016 6th IEEE Inter. Conf. on Biomed. Rob. and Biomech. (BioRob). IEEE, (2016).

19. X. CUI et al. IEEE/ASME Trans. on Mech., (2016).

20. J. LOBO-PRAT et al. Jour. of neuroeng. and rehab., (2014).

21. M.N. Risteiu, M. Leba, O. Stoicuta. Exoskeleton Control System, LAP LAMBERT Academic Publishing, (2020).

22. J. SUN, Y. SHEN, J. ROSEN. IEEE Rob. and Auto. Letters, (2021).

23. C. Rus, N. Negru, P. Patrascoiu, J. Environ. Prot. Ecol., 20 (3), (2019)

24. C. Rus, R. Marcus, L. Pellegrini, M. Leba, M. Rebrisoreanu, A. Constandoiu, IOP Conf. Ser.: Mater. Sci. Eng., 572, (2019)

25. J. GARRIDO, W. YU, A. SORIA. In: 5th IEEE RAS/EMBS Inter. Conf. on Biomed. Rob. and Biomech. IEEE, (2014).

26. C. Rus, N. Mija, M. Leba, WorldCIST 2020, Springer, Cham., (2020)

27. L. ZHOU, Y. LI, S. BAI. Rob. and Auto. Sys., (2017).

28. M. TRÖSTER et al. In: Smart ASSIST. Helmut-Schmidt-Universität, (2018).

29. J. KLEIN et al. In: 2008 2nd IEEE RAS \& EMBS Inter. Conf. on biomed. Rob. and biomech. IEEE, (2008).

30. CHRISTENSEN, Simon; BAI, Shaoping. J. of Mech. and Rob., 2018, 10.4.

31. M.D. Ellis, T.M. Sukal-Moulton, S. Member, J.P.A. Dewald, IEEE Trans. Rob. 25 (2009)

32. P. Langhorne, J. Bernhardt, G. Kwakkel, Lancet 377 (2011).

33. B.H. Dobkin, N. Engl. J. Med. 352 (2005)

34. H.S. Lo, S.Q. Xie, Med. Eng. Phys. 34 (2012)

35. E. VLACHOS, E. JOCHUM, L.Ph. DEMERS. In: 2018 27th IEEE Inter. Symp. on Rob. and H. Interac. Comm. (RO-MAN). IEEE, (2018).

36. Y. MAO, S.K. AGRAWAL. IEEE Trans. on Rob., (2012).

37. F. MISSIROLI et al. Front. in Rob. and AI, (2020).

38. J.L. SAMPER-ESCUDERO et al. IEEE Access, (2020).

39. R. WEI et al. In: 2008 2nd IEEE RAS \& EMBS Inter. Conf. on Biomed. Rob. and Biomech. IEEE, (2008).

40. D. SUI et al. In: 2017 IEEE Inter. Conf. on Adv. Intel. Mech. (AIM). IEEE, (2017).

41. A. FRISOLI et al. Applied Bion. and Biomech., (2009).

42. I. GAPONOV et al. Inter. Jour. of Cont., Auto. and Sys., (2017).

43. S. KIM et al. Applied ergonomics, (2018).

44. A. SCHIELE, G. VISENTIN. In: 7th Inter. Sym. on AI, Rob. and Auto. in Space. (2003).

45. J.P. PINHO et al. In: 2020 42nd Annual Inter. Conf. of the IEEE Eng. in Med. \& Bio. Soc. (EMBC).

IEEE, (2020).

46. T. BUTLER et al. In: ASSE Prof. Dev. Conf. and Expo.. Amer. Soc. of Safe. Eng., (2017). 